Revue des patrimoines

27 | 2015

Le cheval et ses patrimoines (2e partie)

\title{
Deux pieds, quatre sabots. Collecter le présent à l'écomusée du Perche
}

\section{Évelyne Wander}

\section{OpenEdition}

Journals

\section{Édition électronique}

URL : http://journals.openedition.org/insitu/12117

DOI : 10.4000/insitu. 12117

ISSN : 1630-7305

\section{Éditeur}

Ministère de la culture

\section{Référence électronique}

Évelyne Wander, «Deux pieds, quatre sabots. Collecter le présent à l'écomusée du Perche », In Situ [En ligne], 27 | 2015, mis en ligne le 02 novembre 2015, consulté le 03 mai 2019. URL : http:// journals.openedition.org/insitu/12117 ; DOI : 10.4000/insitu.12117

Ce document a été généré automatiquement le 3 mai 2019.

\section{(c) (i) (9)}

In Situ Revues des patrimoines est mis à disposition selon les termes de la licence Creative Commons Attribution - Pas d'Utilisation Commerciale - Pas de Modification 4.0 International. 


\title{
Deux pieds, quatre sabots. Collecter le présent à l'écomusée du Perche
}

\author{
Évelyne Wander
}

Deux pieds, quatre sabots (fig. 1) est le titre d'une publication faisant la synthèse d'une enquête ethnologique et d'un reportage photographique de Christian Malon, éditée à la faveur d'une exposition produite par l'écomusée du Perche, en 2014. Cette présentation mettait en scène des photographies, des objets et des textes contribuant à interroger le compagnonnage de l'homme et des races de chevaux de trait en Normandie et dans le Perche, au XXI ${ }^{e}$ siècle. 
Figure 1

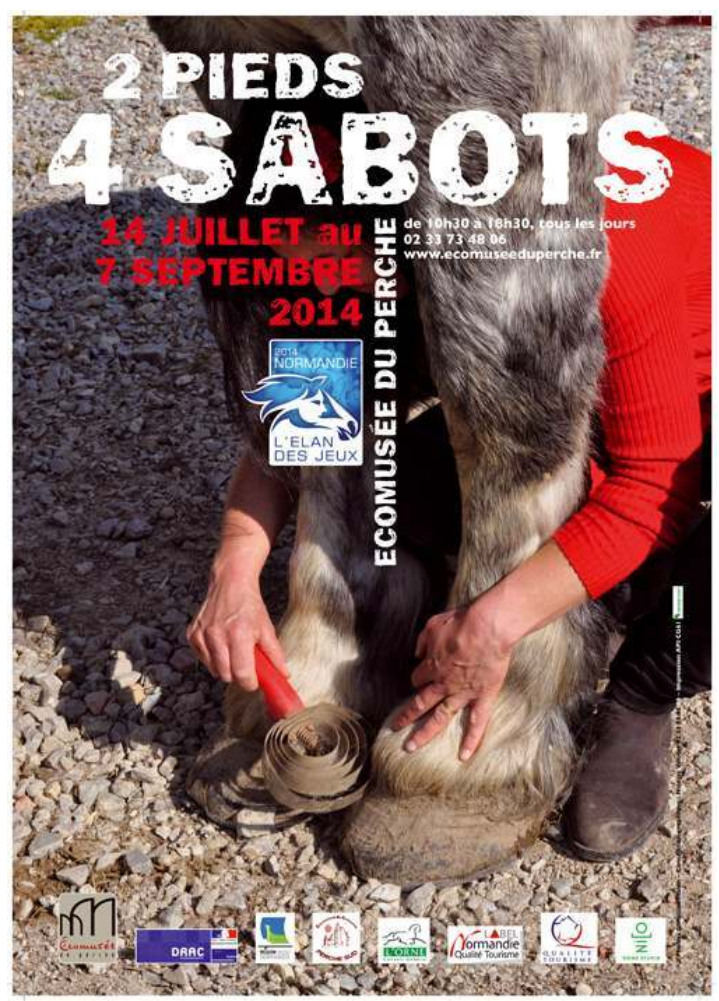

Affiche de l'exposition Deux pieds, quatre sabots.

Phot. Malon, Christian. Graphisme Verrier, Nicolas. @ Christian Malon.

Ce travail met en perspective une mutation remarquable du début de ce siècle : celle du retour du cheval de trait dans des espaces urbanisés pour des usages repensés, symbolisant la recherche d'un nouvel équilibre entre l'homme et la nature. Aujourd'hui, le contexte économique et le développement durable contribuent à relancer, en la diversifiant, l'utilisation du cheval percheron et du cob. Ces nouveaux débouchés sont aussi, pour la filière, accompagnés d'une prise de conscience de la pénibilité du travail pour l'homme et du bien-être animal. Cette actualité permet un retour critique sur la collection du musée et ainsi, de juger des permanences et des innovations dans le domaine du cheval de trait.

Dans le parcours muséographique de l'écomusée (1985), une grande place avait été faite au thème du cheval percheron, mettant en valeur les liens existants entre artisanat et cheval.

Nous avions exposé lors d'un colloque précédent, "Collections et collectionneurs en France et en Europe » (2012), (mettre lien en note) les difficultés théoriques et matérielles inhérentes à une collection concernant le patrimoine lié au cheval de travail et en quoi celle-ci servait la cause d'un patrimoine vivant.

Lors de la création du musée des Arts et Traditions populaires du Perche, en 1972, le deuil de la séparation du cheval au profit du tracteur symbolisait la disparition d'un mode de vie. Le dépôt d'objets pour la collection prenait alors le sens d'une résilience, d'une réparation. Le musée a rempli ce rôle, mais ce lieu de dévotion, par la grâce de la médiation (visites, manifestations, ateliers pédagogiques, expositions, films), a participé dès les années 1990 au soutien d'éleveurs locaux. 


\section{Passer à autre chose}

6 La journée à thème, intitulée "Fête du cheval percheron", est aujourd'hui la manifestation la plus importante de notre établissement. Elle propose, d'une part, des présentations patrimoniales appartenant à la collection du musée ou à d'autres partenaires tels le COMPA ${ }^{1}$ ou encore à des collectionneurs, et d'autre part, la diffusion de connaissances propres à l'élevage, très représentative de notre action. La préparation de la Fête du cheval percheron est conduite comme un atelier pédagogique. Les éleveurs professionnels ont un rôle de conseil auprès des éleveurs amateurs. Un aspect primordial de cette manifestation est l'ouverture sur les utilisations actuelles du cheval : débardage, agriculture biologique, traction en milieu urbain, techniques agricoles transférables vers les pays émergents.

7 C'est précisément lors d'une édition de cette manifestation, dont le thème était l'environnement et les énergies renouvelables, qu'un éleveur amateur, Claude Segaud, a rencontré un meneur qui présentait un bus scolaire hippomobile. "Je me suis dit " pourquoi pas à La-Chaise-Dieu [sa commune] ${ }^{2}$ ? " J'avais 54 ans et je me suis dit que je ferais cela à la retraite ». Cependant, un plan social se prépare dans son entreprise, il saisit l'opportunité et se porte volontaire. Soutenu par le maire de sa commune, M. Dominique René, il élabore son projet.

Cette décision m'a permis de suivre une formation en anticipant ma retraite... J'étais habitué dans mon entreprise précédente à une pression d'enfer, on avait des contrôles de rentabilité. Là, c'est grandiose. Tous les matins j'ai un audit qui se traduit par vingt-quatre gamins, vingt-quatre sourires.

Il conclut : « Les élus ne voient pas toujours que le cheval apporte plus que de l'économie. Le cheval a un rôle social » (fig. 2). 


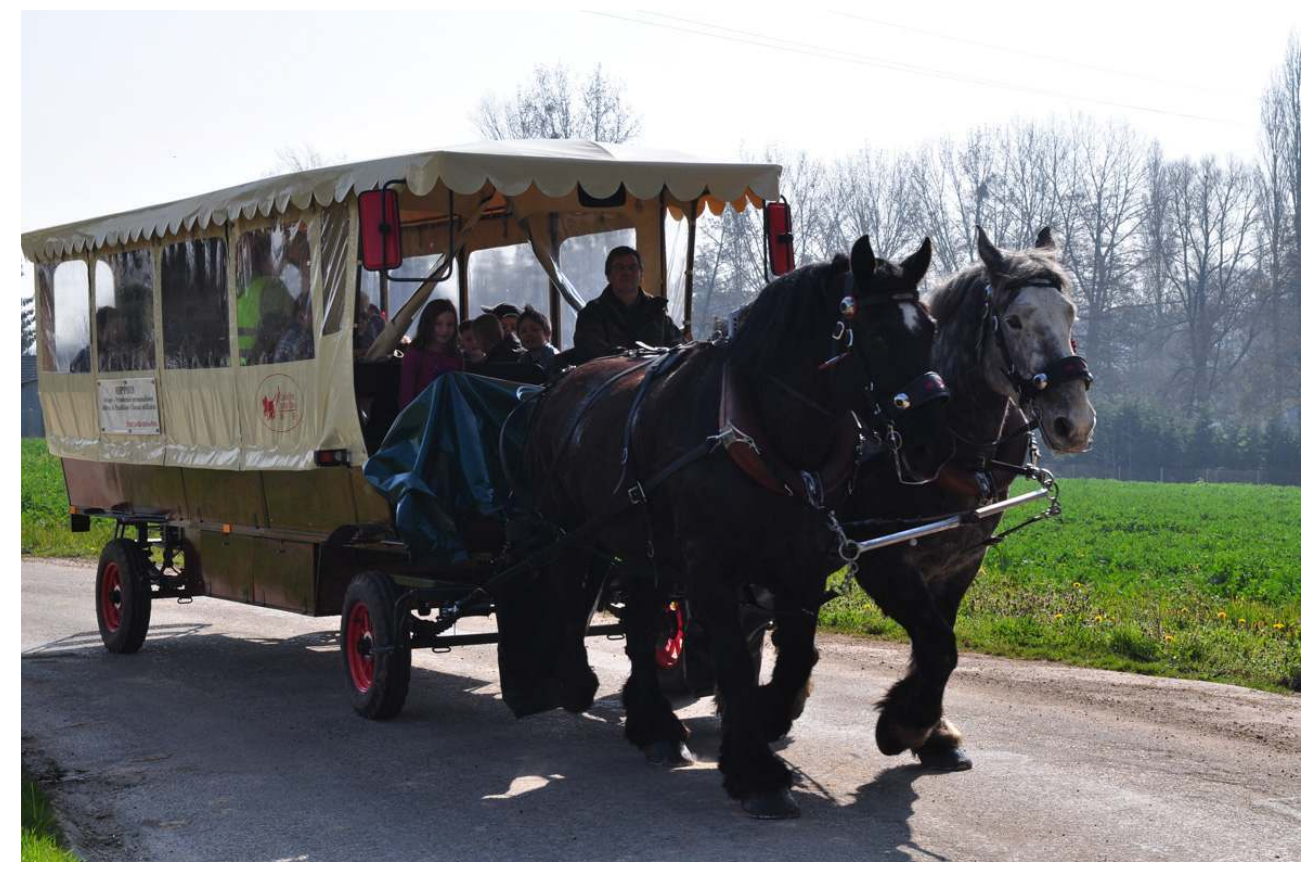

Chaise-Dieu-du-Theil (Eure), ramassage scolaire.

Phot. Malon, Christian. (c) Christian Malon.

Quels ont été les critères qui ont permis la réalisation de ce projet exemplaire ? Claude Segaud connaît le monde du cheval depuis son enfance :

Dès l'âge de 5 ans, je montais à cheval... même si on était mordu, on ne se voyait pas vivre de cette passion. J'ai été technicien dans un groupe de construction de machines destinées à l'imprimerie pendant 28 ans. Parallèlement, j'ai eu des chevaux. Nous avons eu Ondine de La-Chaise-Dieu qui a fait un bon démarrage au salon de l'Agriculture. En 2006, elle a été première diligencière. Cela a contribué à développer davantage le virus, en plus de tous les gens sympathiques que nous rencontrions.

Cette aventure met en évidence le rôle de catalyseur que l'écomusée tient dans le réseau complexe du cheval de travail, celui des apprentissages familiaux, des amitiés, des passions ordinaires ${ }^{3}$, des filières officielles (ministère, salon de l'Agriculture, sociétés hippiques).

11 La mémoire et l'histoire, l'ethnographie et l'ethnologie s'entrecroisent au sein des musées de société afin d'être en prise avec la réalité présente et d'envisager la conservation de ces images instantanées et de ces graphes.

Le travail qui a abouti à Deux pieds, quatre sabots, mené avec Christian Malon, photographe, illustre cette approche, ne serait-ce que par son propre parcours. Christian Malon a construit son premier sujet photographique en comparant les univers paysans auvergnat et normand (1967).

J'ai vécu ces trente dernières années de ruptures du monde agricole avec le sentiment d'abord confus que nous vivions la fin d'un monde ! Mon désir était alors de conserver la mémoire de ces gens, de leurs modes de vie [...] mes photographies je l'espère, au-delà du devoir de mémoire indispensable, peuvent contribuer par leur simplicité et je l'espère par leur vérité, à nous interroger sur notre avenir à 
travers ces regards de paysans, leurs liens avec les animaux et la nature; en gardant l'émotion.

L'enquête consacrée au cheval de trait, en 2014, a fait circuler Christian Malon en Normandie. Si sa carrière a débuté dans un état d'esprit de lutte contre la disparition de la mémoire paysanne, cette nouvelle série lui permet d'observer une campagne différente, celle des mobilités sociales, celle qui vient s'afficher en ville avec de nouvelles valeurs et de nouveaux acteurs.

Citons parmi ceux-ci « Normandie Trait », entreprise de travaux diversifiés qui s'étendent des activités agricoles aux services en milieu urbain. Pour son dirigeant, le cheval est aussi efficace pour la préservation de zones sensibles qu'il peut être une alternative économique, préserver l'environnement en ville, devenir un atout marketing et faire la joie des usagers. Ludovic Eugène crée son entreprise en 2011.

J'ai été formé au haras du Pin. J'ai préparé un diplôme de CS cocher ${ }^{4}$ dans le cadre des formations du ministère de l'Agriculture, grâce à un congé individuel de formation. Auparavant, j'étais conducteur routier, c'est une reconversion. J'ai toujours été passionné par les chevaux de trait. Suite à ma formation, je me suis installé... Le travail, c'est le salut des chevaux de trait. Aujourd'hui, il faut des chevaux polyvalents. Je travaille avec de grandes villes: Angers, Caen, Paris... Je suis régulièrement contacté par les médias...

L'entreprise de trois salariés s'est installée à Brecey (Manche). Sur le fond, Ludovic Eugène souhaite se démarquer d'une attitude tournée vers le passé.

Il existe un décalage générationnel. On ne peut pas vivre en attendant les clients sur la place à 2 euros le tour d'attelage. Le folklore a eu son utilité pour le maintien des races, il faut passer à autre chose. Il faut se placer sur d'autres activités, par exemple les panneaux publicitaires, en ville, sur des attelages.

La diversification de la clientèle permet de remplir le plan de charge.

Il faut au minimum 20 jours de travail par mois et le week-end, c'est du surplus ! Dans mon entreprise, hommes et chevaux ont quelques jours de congés par an et c'est viable. Pour certaines activités comme l'arrosage des fleurs en ville, il faut considérer le tracteur qui roule au pas à la même vitesse que le cheval. Il faut donc viser des choses économiquement réalisables. Il faut travailler les prix pour répondre aux commandes. Il faut pérenniser les activités viables et se fixer un objectif comme un salaire décent. Pour moi, le cheval, je le considère comme un amortissement, aussi je sais combien il coûte par jour.

17 Cet exemple met en évidence le destin commun de l'homme et du cheval, la reconversion professionnelle et la réhabilitation $d u$ "tractionneur ${ }^{5} s^{\prime}$ appuyant sur la réalité du monde agricole contemporain occidental, un monde très mouvant fonctionnant par spécialités à l'instar de la société industrielle qui répond aux besoins du marché.

\section{L'avenir des vieux métiers}

Dans les années 1960, le cheval de trait est devenu inutile dans le nouveau schéma de l'utilisation des campagnes. Il passe du rôle de tractionneur à celui de produit destiné à la boucherie. Il devient officiellement un cheval lourd ${ }^{6}$, qualificatif adapté à la filière hippophagique.

Destin funeste pour les races de trait qui ont contribué à relever l'économie française en 1948. De ce cheptel de 2 millions de bêtes ne subsistent plus que 150000 chevaux dans les années 1980. Pourtant, cette mémoire ne se dissipe pas et croise de nouvelles aspirations : 
la régionalisation, l'environnement avec ses corollaires (paysages, conservation du patrimoine génétique), la démocratisation des loisirs équestres.

En 19947, les races de chevaux lourds redeviennent des races de chevaux de trait. Des aides contribueront à sauver ces races rustiques d'élevage considérées comme races en voie de disparition. L'identité régionale est renforcée et de nouvelles dénominations apparaissent : l'ardennais du Nord devient trait du Nord et le cob prend le titre de cob normand. Les berceaux de race se sont maintenus, mais des zones d'élevage se sont déplacées. De nouveaux besoins émergents coïncident avec la modification sociologique des éleveurs. L'hippophagie, si elle continue d'être pratiquée, est toujours un sujet délicat à aborder avec les éleveurs. Les loisirs, le sport, l'écologie, l'engouement des femmes pour l'équitation ont modifié ces vingt dernières années la relation de l'homme au cheval.

21 Le cheval de trait réapparaît dans les villes et les campagnes pour raisonner l'utilisation systématique de la motorisation, préserver ainsi l'environnement et repenser les sociabilités. Si de nouveaux développements se font jour, ils reposent sur des savoir-faire qui se sont transmis sur la longue durée. La préservation de la mémoire, le recueil de l'histoire et l'engagement des acteurs ont permis de transmettre les outils des mutations présentes.

Il avait été prédit à Daniel Vallée, dans les années 1960-1970, que la maréchalerie n'était pas un métier d'avenir. Il a ainsi été imprimeur pendant 23 ans, alors que depuis son enfance, il avait la passion des chevaux. Parallèlement à son métier, il a commencé à pratiquer des activités liées au cheval. La passion du cheval l'a emporté, il crée son entreprise en 1994 (fig. 3).

J'ai environ 200 clients, des particuliers, et parmi ceux-ci de petits clients. Je ferre 100 percherons, à l'anglaise, 200 ânes, le reste en chevaux de selle... Dans $60 \%$ des cas je m'occupe seul du cheval, je ne vois pas le client car les uns travaillent, les autres sont résidents secondaires.

Figure 3

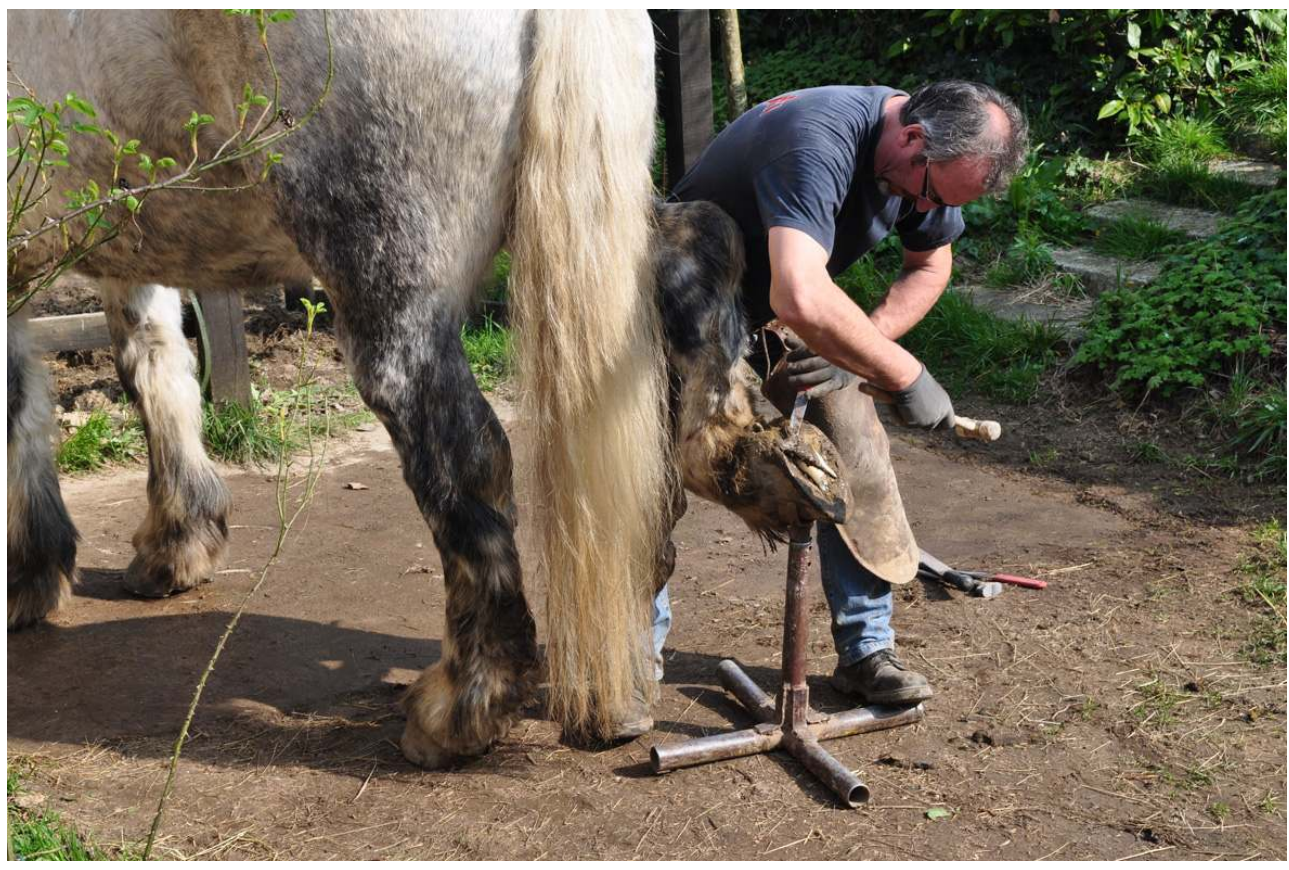

Perche (Orne), maréchal-ferrant.

Phot. Malon, Christian. (C) Christian Malon. 
23 À l'instar du métier de maréchal-ferrant, ce qui était pensé comme disparu et portant le nom générique de vieux métiers renait dans un autre contexte, sous d'autres formes.

Cette nouvelle donne remet en cause l'héritage des musées d'Arts et de Traditions populaires. Si le vieux est actuel, alors que conserver? En quête de ce qui disparaissait, les musées de société, les écomusées, héritiers de l'engouement prolifique des collections d'arts et de traditions populaires, sont confrontés à l'analyse du temps présent et à l'explosion du concept ethnographique basé sur un territoire, un groupe, un temps donné, une culture. L'activité liée au cheval de trait illustre cette complexité.

Les éleveurs, les agriculteurs (fourniture de fourrages), les métiers de service tels que les cochers, les palefreniers-soigneurs, les vétérinaires, les techniciens de matériels hippomobiles, les bourreliers, les formateurs, les personnels administratifs sont autant de spécialités participant de la filière du cheval de trait. La traction animale est potentiellement un moteur économique et sociétal.

Depuis le premier congrès des Chevaux territoriaux, à Trouville, en 2003, la question du cheval en ville est devenue un sujet qui dépasse le stade de l'anecdote. Un rapport récent du ministère de l'Agriculture ${ }^{8}$ comptabilisait plus de 200 villes ayant choisi d'utiliser des équidés pour régler un ou plusieurs problèmes environnementaux ou sociaux : l'entretien des espaces verts et des zones sensibles, les navettes, le ramassage scolaire, les circuits touristiques, les manifestations diverses, la collecte des déchets, les projets pédagogiques et la médiation sociale.

Le directeur général des services de la Ville de Trouville, Olivier Linot, a conçu le projet, en 2000, de gérer l'enlèvement des déchets en ayant recours à la traction animale dans une ville touristique qui passe de 5000 habitants en semaine à 20000 en fin de semaine. La conséquence de l'activité de restauration se traduit par cinquante tonnes de verre par an, à collecter dans des rues piétonnes étroites où la circulation des véhicules est assez dense. Après étude, la Ville acquiert un percheron et une charrette. Elle recrute quatre personnes dans le cadre des contrats Emploi jeune. Au départ, l'esprit de bonne gestion a présidé à cette opération, considérant le prix de revient nettement inférieur à l'amortissement d'un camion et aux frais de fonctionnement inhérents. Certains administrés étaient réticents, arguant «le retour en arrière». Ils sont aujourd'hui conquis. La présence d'un attelage en ville contribue également à ralentir le flux automobile. Côté écuries, cob et percheron, les deux grands types de trait normands sont représentés. Côté équipe, les Trouvillais côtoient Marie, Pascal, Adèle (apprentie) et Christelle, laquelle a pu trouver sa voie grâce à l'initiative de la Ville de Trouville.

Je suis cavalière depuis l'âge de huit ans. Le milieu du cheval, c'était difficile de penser que ce serait mon métier, on n'imaginait pas ce genre de travail. Je n'avais pas envie de faire des box tous les jours. C'est trop dur physiquement. Ici, j'ai pu allier la passion du cheval avec le confort de la fonction publique (fig. 4). 
Figure 4

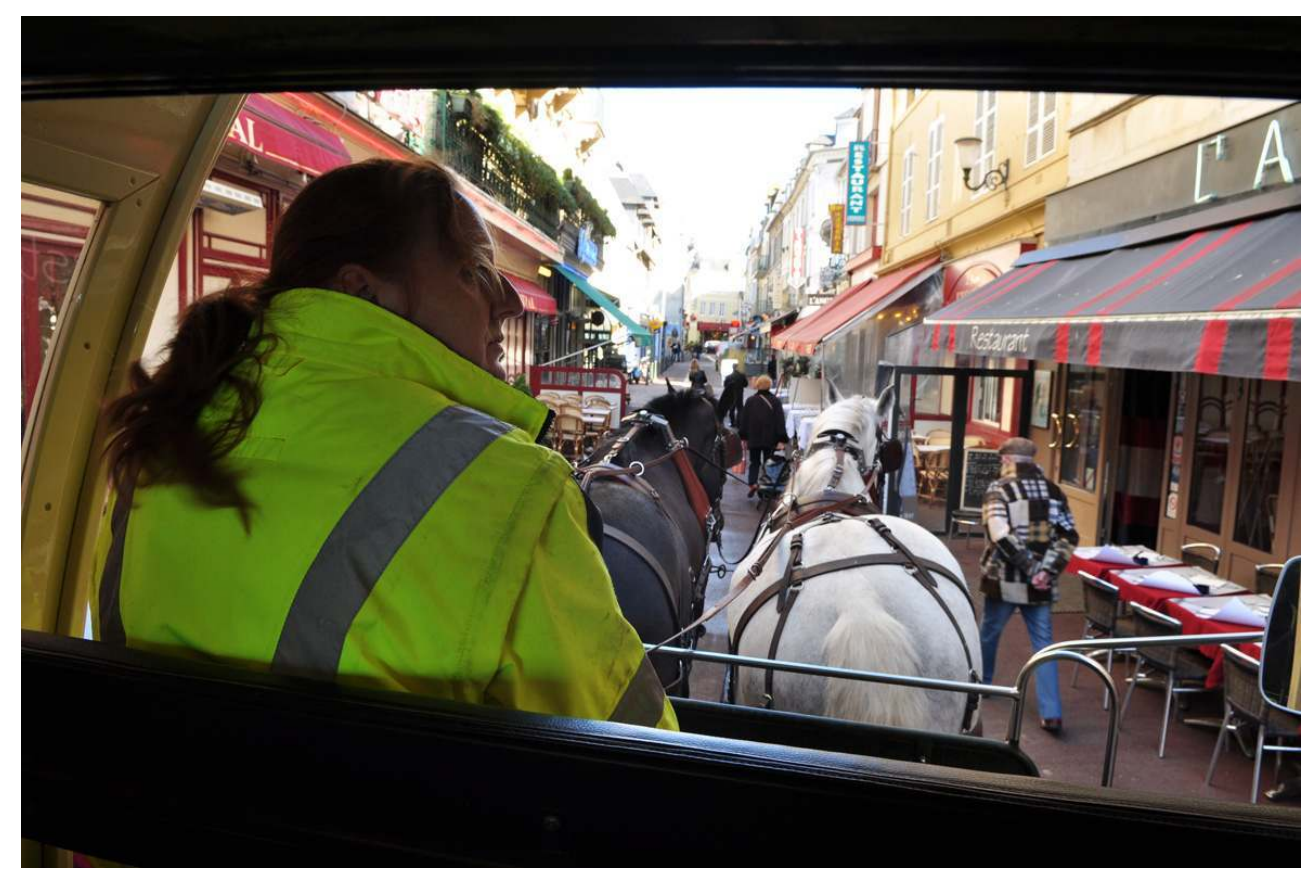

Trouville-sur-Mer (Calvados), employée municipale de la voierie.

Phot. Malon, Christian. (c) Christian Malon.

On note que les quelques lignes du curriculum vitae permettant généralement à l'employeur de cerner la personnalité d'un candidat sont ici primordiales. Les loisirs sont là une source de recrutement, changement notable de registre dans une société en crise qui ouvre des perspectives nouvelles également aux femmes.

\section{Trait familier, trait productif}

Après la Seconde Guerre mondiale, le nombre de cavaliers se limitait à quelque 30000 licenciés. Aujourd'hui, près de 600000 cavaliers font de la Fédération française d'équitation la troisième fédération sportive de France. La démocratisation de cette pratique en a favorisé l'accès aux femmes depuis les années 1960. Elles représentent actuellement les trois quarts des effectifs et participent largement à l'encadrement, aux animations, à la gestion des centres équestres. Cette transformation profonde des mentalités a permis aux femmes de se projeter et de penser une activité équestre comme accessible. Les chevaux de trait font partie de ces possibles, que ce soit dans le cadre professionnel ou amateur.

Valérie a grandi dans une ferme entre L'Aigle et Mortagne. Son voisin élevait des chevaux percherons.

C'est là que j'ai développé mon amour du cheval percheron. Je suis issue d'une famille nombreuse, mes parents n'avaient pas les moyens de me payer des cours d'équitation, aussi lorsque j'ai pu travailler à 18 ans, j'ai pris des cours.

31 Valérie achète ensuite un percheron diligencier.

Quand j'ai vu Qésako, j'ai eu le coup de foudre. C'est une histoire d'amour entre ce cheval et moi, nous avons une relation de complicité, on fait des petits tours de cirque. Il faut être doux avec eux, ils nous le rendent bien. 
Valérie prépare de petits spectacles afin de faire travailler son cheval ; ceux-ci servent de lien et connectent le monde du cheval de trait à la société :

Je pratique la zumba', c'est pour cela que j'avais essayé en 2013 de rapprocher deux univers, celui de la danse et celui du percheron. C'était une touche nouvelle pour rapprocher les gens. J'ai passé un bon moment.

Elle cherche à conjuguer ses aspirations et propose une autre image du cheval percheron. "C'est un côté féminin qui ressort, un message d'amour, un message de paix ».

Ainsi, le monde du cheval de trait est-il transformé par des utilisateurs qui modifient ses images et ses codes. Cette proximité nouvelle de la société rend complexe la relation de l'homme avec le cheval et nombre de débats ont cette polémique pour centre: le cheval, du bien meuble à l'animal familier. Pour les éleveurs, si le bien-être animal est pour le plus grand nombre une valeur essentielle, la question primordiale est avant tout celle de la continuité de leur activité.

Pour le muséologue, une belle vitrine et des cartels sont insuffisants pour appréhender ces réalités sociales. L'approche chronologique était (ou est encore) la mission suprême du musée afin de faire mesurer au public, au citoyen, les progrès de la société. Quelle mission le musée a-t-il aujourd'hui si ce n'est d'aider les visiteurs à aborder des mondes inconnus, pourtant si proches? C'est le cas pour l'agriculture et l'élevage équin, lequel n'échappe pas à cet état de fait. La part de l'individualité transforme la production en projets plus personnels, en rupture avec la génération précédente et puisant dans des représentations plutôt que dans une transmission par filiation, difficilement transférables sur un autre type d'exploitation.

Jean-Boris est installé en agriculture « bio » avec des vaches laitières.

Le métier est un choix, les chevaux je ne sais pas d'où ça vient... Bien avant mon installation, j'ai eu ma première jument à 14 ans. C'était déjà un cob... J'ai appris à utiliser la charrue brabant avec deux anciens, par hasard, lors d'un concours. J'ai remplacé un vieux monsieur.

Membre actif de France Trait, Jean-Boris regarde avec objectivité les possibles usages du cheval de trait aujourd'hui. L'utilisation du cheval en exploitation laitière ne lui semble pas viable.

Il y a un rapport à faire entre l'effectif du troupeau et la surface. J'ai quarante-cinq vaches, c'est impossible de suivre avec les chevaux. Pour le maraîchage ou la vigne sur quelques hectares, c'est valable et on peut faire vraiment un parallèle avec le prix du tracteur.

L'adaptation de l'outillage est indispensable. Il existe de plus en plus de fournisseurs en France et à l'étranger. Le plus souvent, il s'agit de matériels adaptés aux besoins spécifiques du client. Un parallèle peut être fait avec l'essor du tracteur en France dans les années d'après-guerre, pendant lesquelles de petits artisans mécaniciens sortaient des modèles à valeur de prototype (fig. 5). 


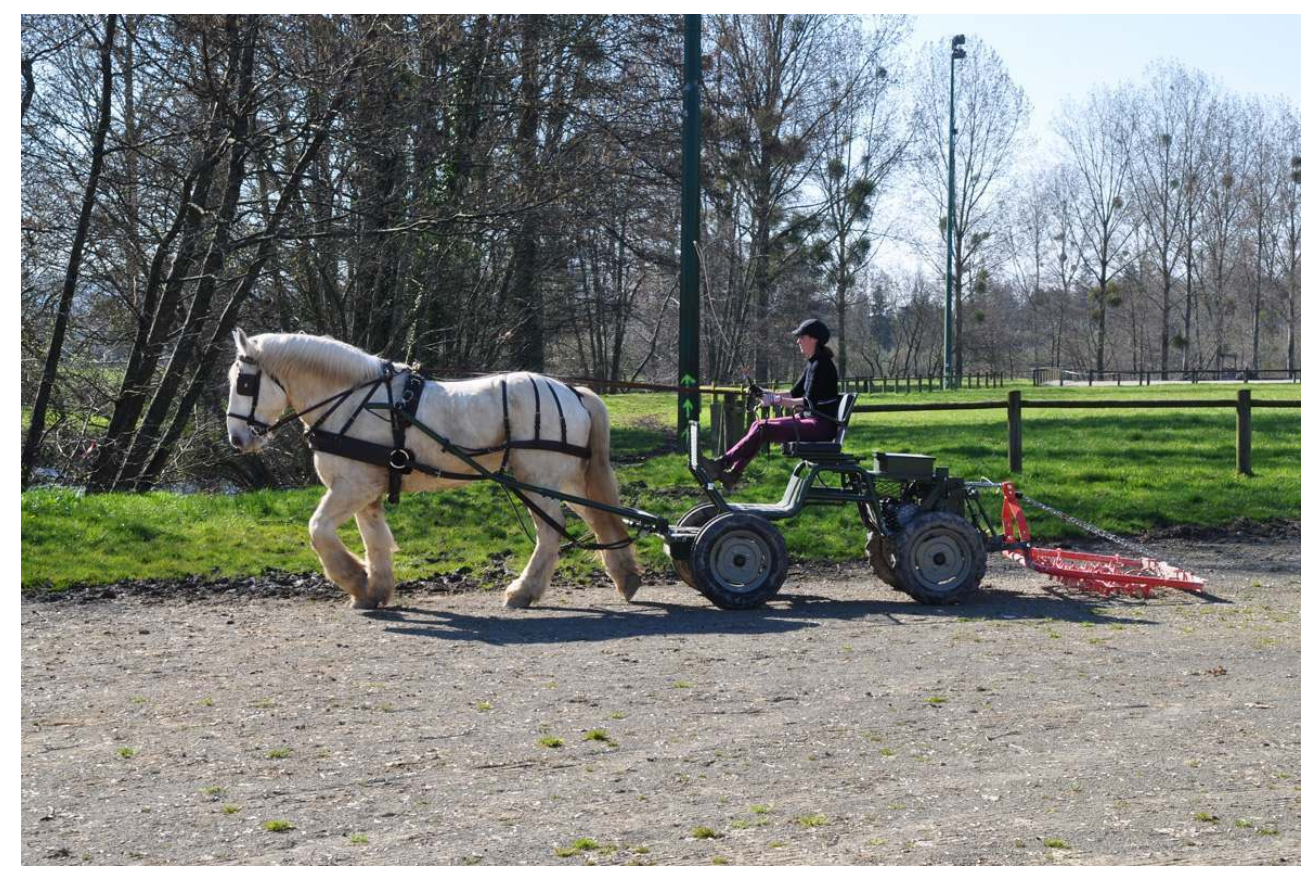

Manche. Normandie Trait, porte-outils Tractozore (entreprise Clémobil) et herse.

Phot. Malon, Christian. (c) Christian Malon. composites, la fourniture d'avant-trains polyvalents, la prise en compte des milieux, l'adjonction de motorisation électrique afin de répondre aux objectifs de réduction de la pollution, de la pénibilité du travail pour l'homme et le cheval, de réduction des temps de travail et donc des coûts. Jean-Boris fabrique son outillage lui-même. «Je sais souder et meuler, j'essaie toujours d'avoir un coût moindre ».

La structure de l'exploitation conduit Jean-Boris à pratiquer des activités très diversifiées.

Je fais du débardage sur mes terres, je refais des prairies au printemps. Il ne faut pas mentir aux gens car ce n'est pas très rentable. Moi, je me sers de ces travaux pour dresser mes chevaux. Je suis naisseur, éleveur, dresseur... L'élevage, c'est un métier, sélectionner c'est un métier. Une race ne peut perdurer dans le cadre unique du loisir. Il faut consolider les éleveurs agriculteurs. Le désengagement de l'État dans le soutien à l'élevage déstabilise la filière... Les nouveaux services sont de petits espoirs. On peut penser que le prix de l'essence laisse la place à une énergie alternative et que les loisirs participent aussi à ce déploiement. L'élevage de chevaux de trait, c'est quelque part du militantisme. Pendant cinq ans nous avons lutté (France Trait) pour que le terme herbivore entre dans la PAC ${ }^{10}$ mais cela n'a pas été retenu. On aurait gagné sinon.

41 Le témoignage de cet éleveur permet de considérer la question du cheval et de ses patrimoines dans une dimension d'économie internationale et d'enjeux de société dont le musée doit rendre compte.

\section{Le moteur d'utopie}

42 L'organisation de l'exposition « Deux pieds, quatre sabots » a permis d'examiner la réalité du cheval utilitaire en Basse-Normandie, donnant un aperçu des nouveaux usages du 
cheval de trait et de ses acteurs, hommes et femmes, qui exercent un métier de passion ou vivent un loisir engagé.

Les territoires normands et percherons ont conservé une mémoire des chevaux de trait. Le percheron et le cob n'ont pas disparu des imaginaires, ils suscitent encore des projets et demeurent inscrits dans la construction de la vie sociale et économique. Toujours sousjacent, ce mouvement de fond a resurgi pour s'ancrer là où il était le moins attendu. La ville pour horizon, le ratio économique pour cadre, ont étoffé de nouvelles compétences. Le cheval œuvrait jusque-là, plus discrètement, à la protection des espaces sensibles et à l'agriculture biologique, là où il paraissait le plus légitime. Plus étonnant encore, il devient une passerelle entre milieux, entre univers, il contribue à créer des liens sociaux.

Ce moteur d'utopie entraîne des reconversions professionnelles, des modifications dans la perception du monde et les comportements. Dans les rues de Trouville, on patiente désormais derrière la voiture hippomobile.

La relégation du cob ou du percheron au magasin des accessoires folkloriques a pu être atténuée par l'arrivée de nouvelles générations qui considèrent le progrès avec circonspection, de nouveaux acteurs qui réhabilitent ces laissés pour compte de l'industrialisation de l'agriculture, des personnes qui s'identifient à cette marginalisation pour la combattre. Devenue transversale, cette affaire du cheval de trait s'est installée comme une potentialité, non plus comme une solution aberrante, sur le bureau du politique et de l'administratif, dans le curriculum vitae du demandeur d'emploi et au cœur d'un choix de vie.

Dans le corpus des documents anciens, rares étaient les photographies qui représentaient des femmes en situation d'éleveur ou d'utilisateur d'un cheval de trait.

Les clichés de Christian Malon illustrent la modification de ces milieux, dont l'implosion a provoqué le renouvellement des mœurs, des pratiques et des représentations.

Si parfois le cheval de trait fait spectacle, il ne fait pourtant pas toujours recette pour ses éleveurs. Concernant la PAC, France Trait, fédérant les associations des neuf races de chevaux de trait, demande la reconnaissance de la filière équine comme un acteur agricole à part entière et réclame des aides directes. Valorisation des herbages, des savoir-faire, revitalisation des zones rurales, le cheval de trait demeure un enjeu pour l'avenir des campagnes.

49 Concernant les collections matérielles du musée, la phase de réappropriation d'un outillage différent, proche de l'expérimentation, demande une veille attentive. La présente exposition en sera la phase exploratoire.

50 Ces nouvelles pistes de recherche et de collectage devront articuler patrimoine immatériel et matériel. La création de groupes pluridisciplinaires est indispensable pour aborder ces questions. Le monde du cheval de travail a vécu plusieurs mutations majeures, échappant de peu à la disparition. Ses oripeaux étaient conservés au musée avec leurs cortèges d'autrefois et de vieux métiers. Parions que le collectage de l'actualité du cheval de trait, faite de passion et d'innovation, permettra aux musées qui ont la charge de ce patrimoine de se renouveler également et de s'appuyer en premier lieu sur la conservation du patrimoine immatériel. 


\section{BIBLIOGRAPHIE}

\section{Sites et articles}

Note juridique sur la proposition de loi déposée par M. Luca, député des Alpes-Maritimes, modifiant le statut juridique du cheval d'animal de rente en animal de compagnie. Rédacteurs : Maître Guillaume Fallourd, consultant GESCA et Xavier Bonté, responsable du département juridique France-Galop : http://www.france-trait.fr/userfiles/5/File/note-gesca-2010--2.pdf.

À propos de la proposition de loi visant à changer le statut du cheval d'animal de rente à animal de compagnie, Friday, May 7th, 2010 : http://www.chevaldetrait68.org/.

TSAAG-VALREN, Amélie. « Le cheval, animal de compagnie ? » Nouveau débat, n44, juin 2013 : http://www.cheval-savoir.com/equipe-cheval-savoir.

Le-cheval-animal-de-compagnie-une-nouvelle-proposition-de-loi : http://www.chevalmag.com/ Actualites/Divers/

En Basse-Normandie, ils valorisent le cheval de trait par le travail :

http://www.entreprises.ouest-france.fr/node/115591

Un Forum des Métiers avec Normandie Trait. Publié par JLD.

L'énergie animale en quelques traits, brochure éditée par France Trait, avec le soutien financier du Fonds Éperon, 24 juin 2013.

\section{Rapports et documents}

Note juridique sur le cheval, « animal de compagnie ». Institut du droit équin, hôtel Burgy - 13, rue de Genève, 87100 LIMOGES, étude diligentée par M. Lebrun en 2010 (Les enjeux et les perspectives de la filière équine en France. Avis et rapports du conseil économique, social et environnemental de la République française).

Proposition de loi visant à modifier le statut juridique du cheval en le faisant passer d'animal de rente à animal de compagnie, $\mathrm{N}^{\circ}$ 1191, ASSEMBLÉE NATIONALE, Enregistré à la Présidence de l'Assemblée nationale le 26 juin 2013.

LANGLOIS, Bertrand. La génétique et la sélection du cheval percheron. Institut National de la recherche Agronomique (INRA), 78352 Jouy-en-Josas (France).

«L'environnement entre nature et paysage. Sud-Ouest Européen ». Revue géographique des Pyrénées et du Sud-Ouest, $n^{\circ}$ 7, mai 2000. Toulouse : Presses univ. du Mirail, 2000.

\section{Ouvrages}

DAL'SECCO, Emmanuelle. Les chevaux de trait. Paris : Artemis éditions, 2006.

MAVRÉ, Marcel. Attelages et attelées : un siècle d'utilisation du cheval de trait. Paris : Campagne et compagnie, 2011.

\section{NOTES}

1. - Conservatoire de l'agriculture, Chartres (Eure-et-Loir). 
2. - Chaise-Dieu-du-Theil est une commune rurale de 245 habitants permanents, située dans le département de l'Eure (Haute-Normandie).

3. - BROMBERGER, Christian (dir.). Passions ordinaires. Du match de football au concours de dictée. Paris : Bayard, 1998.

4. - Préparation du certificat de spécialisation agricole option "utilisateur de chevaux attelés » (CS UCA). Diplôme de niveau V délivré par le ministère chargé de l'Agriculture. Le but : acquérir une qualification professionnelle reconnue dans le domaine de l'attelage mobilisant des véhicules hippomobiles à des fins touristiques ou utilitaires, ou dans le domaine du travail agricole.

5. - Terminologie adoptée par MAVRÉ, Marcel. Attelages et attelées : un siècle d'utilisation du cheval de trait. Paris : Campagne et Compagnie, 2011.

6. - Décret $\mathrm{n}^{\circ}$ 76-351 du 15 avril 1976 rendant applicables aux équidés les articles 2, 3, 7, 10-1, 10-2, 10-3 et 16 de la loi ${ }^{\circ}$ 66-1005 du 28 décembre 1966 sur l'élevage.

7. - JORF $\mathrm{n}^{\circ} 78$ du 2 avril 1994, Arrêté du 11 mars 1994 portant modification de l'arrêté du 23 juillet 1976 relatif aux races reconnues et aux appellations des chevaux nés en France.

8. - Rapport du ministère de l'Agriculture, CGAAER, septembre 2012.

9. - La zumba est un programme d'entraînement physique combinant des éléments d'aérobic et de la danse jazz. Les chorégraphies s'inspirent des danses latines mais aussi de styles variés comme le Bollywood, la danse du ventre ou le swing.

10. - La politique agricole commune (PAC) est la plus importante politique de l'Union européenne mais ce budget est en baisse. Ses buts : la productivité, un revenu équitable aux agriculteurs, la stabilité des marchés, la sécurité alimentaire, des prix raisonnables pour les consommateurs. Les principes de respect de l'environnement et de développement rural se sont ajoutés récemment.

\section{RÉSUMÉS}

Deux pieds, quatre sabots est le titre d'une publication faisant la synthèse d'une enquête ethnologique et d'un reportage photographique de Christian Malon, éditée à la faveur d'une exposition présentée en 2014 à l'écomusée du Perche. Elle met en scène des photographies, des objets et des textes contribuant à interroger le compagnonnage de l'homme et des races de chevaux de trait en Normandie et dans le Perche, au xxI ${ }^{\mathrm{e}}$ siècle. Ce travail scrute une mutation remarquable du début de ce siècle, celle du retour du cheval de trait dans des espaces urbanisés pour des usages repensés, symbolisant la recherche d'un nouvel équilibre entre l'homme et la nature. Aujourd'hui, le développement durable contribue à relancer, en le diversifiant, l'élevage du cheval percheron et du cob. Ces nouveaux débouchés sont aussi, pour la filière, accompagnés d'une prise de conscience de la pénibilité du travail pour l'homme et du bien-être animal. Cette actualité permet un retour critique sur la collection du musée, permettant de juger des permanences et des innovations dans le domaine du cheval de trait. Les processus de collectage du professionnel de musée et ses modes de restitution sont-ils adaptés à ces réalités nouvelles?

Two feet, four hooves, collecting the present at the Perche écomusée. Deux pieds, quatre sabots (two feet, four hooves) is the title of a book resulting from an ethnological investigation and a photographic reportage by Christian Malon, published to coincide with an exhibition put on in 2014 by the Perche ecomuseum. It presents photographs, objects and texts which seek to question the company between men and different races of Normandy draft horses, in the Perch region, at the beginning of the twenty-first century. It is a piece of work that documents a 
remarkable change witnessed during this period in which the horse is making a comeback in urban spaces and for new uses, symbolising the quest for a new equilibrium between man and nature. Sustainable development has encouraged and diversified the use of Percheron and Cob horses. These new uses are accompanied by a new awareness of the difficulty of the work for men and of the animal's well-being. This very contemporary observation throws new light on the museum's collection, showing up permanent and novel features in this realm of the draft horse. But are the museum curator's usual processes for collecting and displaying objects appropriate for these new realities?

\section{INDEX}

Mots-clés : cheval de trait, musée d'ethnologie, patrimoine immatériel, Basse-Normandie, Perche

Keywords : draft horse, ethnological museum, intangible heritage, Basse-Normandie, Perche

\section{AUTEUR}

\section{ÉVELYNE WANDER}

directeur et responsable scientifique de l'écomusée du Perche, ethnologue direction@ecomuseeduperche.fr 